\title{
A Case Study on Offshore Platform Deformation Monitoring by using InSAR
}

\author{
Amir Sharifuddin Ab Latip ${ }^{1, *}$, Abd Nasir Matori ${ }^{1}$, and Anuphao Aobpaet ${ }^{2}$ \\ ${ }^{1}$ Department of Mechanical Engineering, Universiti Teknologi PETRONAS, Seri Iskandar, Malaysia \\ ${ }^{2}$ Department of Civil Engineering, Kasetsart University (KU), 50 Thanon Ngam Wong Wan, \\ Khwaeng Lat Yao, Khet Chatuchak, Bangkok, Thailand
}

\begin{abstract}
The Interferometric Synthetic Aperture Radar (InSAR) technique is well established technique for onshore applications such as landslide, mining, volcano, urban and dam deformation monitoring. However, the ability of the InSAR technique for the offshore application particularly on the oil platform deformation monitoring is still a pending question. Therefore, a study needs to be conducted in order to assess the feasibility of the InSAR technique for monitoring the deformation of offshore platform. In this study, an advanced InSAR technique called Stanford Method of Persistent Scatterer (StaMPS) was utilized to process a stack of TerraSAR-X data. A mask has been developed as an additional module in the StaMPS to remove the noise on the ocean and relax the assigned condition imposed on the processing parameters. The results show that the number of persistent scatterer (PS) points on the offshore platform increased from 15 to 22 points and a smoother pattern of the velocity estimation could be seen when masking was applied to identify the deformation. Furthermore, some limitations and possible solutions of the PSI technique on the offshore platform application are summarized in this paper to guide future users on this application.
\end{abstract}

\section{Introduction}

Offshore platforms have become important assets for oil and gas industries as the demand for energy from fossil fuel keeps on increasing. Deformation of offshore platforms is one of the major issues concerning the offshore structural integrity and it can be occurred caused by various factors of both natural processes and human activities, including ageing, extreme weather, shallow gas blowout, geotechnical hazards, fatigue, corrosion, dropped heavy objects, seabed instability and helicopter/ship collision impact [1]. Disruption of production or even posing risks to loss of life, loss of assets and environmental consequences may potentially result from the deformation of offshore platforms. Thus, monitoring the deformation of offshore platforms is very crucial as a mitigation effort for unforeseen circumstances.

\footnotetext{
* Corresponding author: asharifuddin2@gmail.com
} 
Ground-based techniques, such as precise levelling, total stations, global navigation satellite system (GNSS) are widely applied in geomatics fields for oil and gas structures deformation monitoring [2 - 4] while accelerometers, piezometers, inclinometers, tiltmeters, extensometers and fiber-optics are mainly applied in geotechnical fields [5 - 7]. Although the ground-based techniques can provide deformation information with high accuracy and reliability, they only provide deformation information at specific observation points with a large investment in logistics cost, human resources, special equipment, and time consumption [8]. Satellite-based InSAR is an invaluable technique which can be used to remotely monitor structure deformation since it does not require direct contact with the monitored structures. Through the development of multi-temporal InSAR techniques, it has a high sensitivity to detect even a small deformation of structures over a long time span with millimeter accuracy [9]. Furthermore, the relatively high spatial and temporal resolutions of current radar satellites, such as TerraSAR-X or COSMO-SkyMed (X-band satellites), allow the identification of a high density of measurement points and frequent surveillance of individual structures [10]. Furthermore, the deformation results of the monitored structures can be further analyzed as a key data source for early warning systems which abnormal or excessive movement indicating potential hazards that require detailed field inspections and preventive measures. Moreover, a large number of observations used and long-term surface deformation monitoring derived from InSAR is a very helpful tool to support the development of reliable deformation models [11 -12].

This study has focused on extracting the surface deformation over a fixed offshore platform by generating a deformation velocity map and displacement time series from the Stanford Method of Persistent Scatterer (StaMPS) technique. The StaMPS technique has been applied on a set of TerraSAR-X images acquired from August 2012 to April 2013 over the offshore platform. This study has developed a mask to exclude the de-correlated area caused by the ocean and left only the monitored offshore platform area to be involved in the processing. This has reduced the number of noisy persistent scatterer (PS) points and made the selection of the PS points more reliable. The limitations and solutions of the Persistent Scatterer Interferometry (PSI) technique on the offshore platform deformation monitoring are also discussed in this paper.

\section{Methodology}

This section will explain the methodology involved in this work:

\subsection{Test Site}

The P platform is a fixed Jacket-Type platform that functions as drilling, production and accommodation (see Fig. 1). It was installed in the South China Sea in 1976 and has been operated for more than 30 years. The platform is situated about $250 \mathrm{~km}$ from the east coast of Kuala Terengganu in a water depth of $74.70 \mathrm{~m}$. This platform consists mainly of three parts: a deck/topside, a jacket and piles. The deck is fixed upon the jacket and is composed of a drilling rig, power generator, production facilities, crew quarters and a helipad. It has a weight of 11,397 tons, a length of $58.9 \mathrm{~m}$ and a width of $31.40 \mathrm{~m}$. Meanwhile, the jacket has eight legs with a height of 85.60 m supported by 12 driven piles into the seabed. The P platform experienced a shallow gas phenomenon in 2003. Since then, field surveys have been carried out to monitor the deformation of the $\mathrm{P}$ platform in an attempt to prevent possible hazards. 


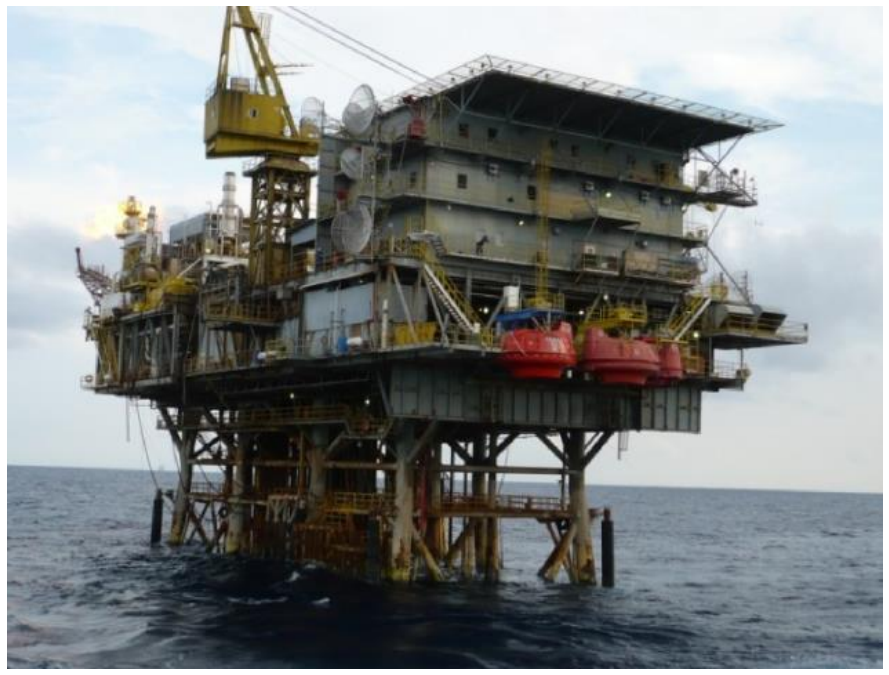

Fig. 1 P platform (Source: [13])

\subsection{Dataset}

A dataset consisting of 11 StripMap images of the TerraSAR-X were acquired over the $\mathrm{P}$ platform at around 6.52 am on each acquisition day along a descending satellite track with an average incidence angle of 26.36 degrees, covering the time period between August 2012 and April 2013. The minimum and maximum intervals between the two successive acquisitions were 11 and 33 days, respectively. The scene on $04^{\text {th }}$ January 2013 was selected as the temporal reference, maximizing the overall coherence of all of the scene pairs. All of the temporal baselines were shorter than 132 days and the perpendicular baselines were shorter than $350 \mathrm{~m}$. The details of the data can be found in Table 1 .

Table 1. Image number, date of the data acquisition and perpendicular and temporal baselines with respect to the master image.

\begin{tabular}{cccc}
\hline Image number & Date $(\mathbf{d d} / \mathbf{m m} / \mathbf{y y y y})$ & $\begin{array}{c}\text { Perpendicular } \\
\text { baseline }\left(\boldsymbol{B}_{\text {eerp }}\right) \\
\text { [meters] }\end{array}$ & $\begin{array}{c}\text { Temporal } \\
\text { baseline }\left(\boldsymbol{B}_{\text {temp }}\right) \\
\text { [days] }\end{array}$ \\
\hline 1 & 25.08 .2012 & 167.8 & 132 \\
2 & 27.09 .2012 & 180 & 99 \\
3 & 30.10 .2012 & 344.5 & 66 \\
4 & 21.11 .2012 & 75.07 & 44 \\
5 & 13.12 .2012 & 180.2 & 22 \\
6 & 24.12 .2012 & 39.32 & 11 \\
\hline 7 & 04.01 .2013 & 0 & 0 \\
\hline 8 & 26.01 .2013 & 4.939 & 22 \\
9 & 17.02 .2013 & 2.715 & 44 \\
10 & 22.03 .2013 & 270.3 & 77 \\
11 & 24.04 .2013 & 63.7 & 110 \\
\hline
\end{tabular}

\subsection{Masking Technique Development and StaMPS Implementation.}

StaMPS software uses the DORIS software to generate interferograms from single look complex (SLC) images [14]. Masking is an additional module proposed in this study which is applied before the step of persistent scatterer candidates (PSC) selection. StaMPS 
software is then used to generate the deformation velocity map and deformation time series [15 - 16]. Fig. 2 shows the processing flow chart of the StaMPS technique.

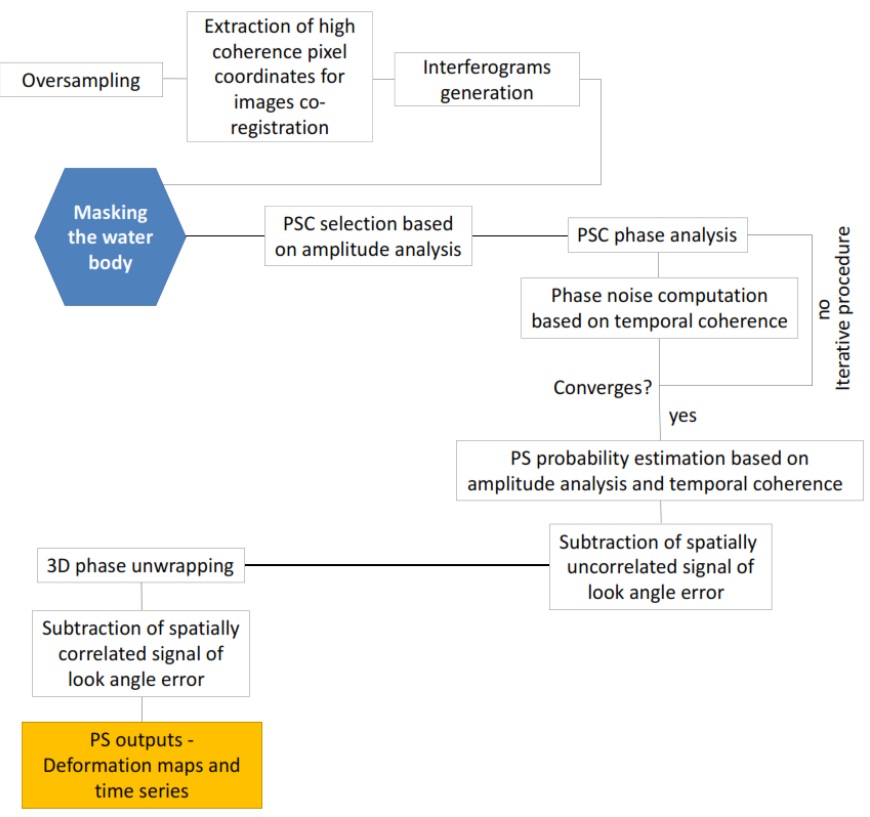

Fig. 2 Processing flow chart of the StaMPS technique. The blue box denotes the new module proposed in this study.

\subsubsection{Reading, Cropping, Oversampling and Master Image Selection.}

The processing begins by reading the input data of SAR images in the SLC format. Then, the SAR images were cropped to the interest area/structures by sizing the window of the master image with dimensions on the order of 200 lines x 200 pixels. After cropping the SAR images, they were oversampled by a factor of 2 in the azimuth (line) and range (pixel) directions. An optimal master image was chosen based on perpendicular and temporal baselines as well as Doppler shift in order to maximize the coherence in the series of interferograms.

\subsection{Co-registration using fc_pos.in file}

Co-registration between the master and slave image is critical for the P platform as the platform has a small size of $30.73 \mathrm{~m}$ (width) $* 58.81 \mathrm{~m}$ (length) and is surrounded by the vast ocean that is affected by temporal de-correlation. The oversampled images were the input for the co-registration of the master and the slave images. The co-registration procedure typically consists of two steps which are coarse co-registration and fine coregistration. After the co-registration, the resample is performed which all the slave images are interpolated to the grid of the master image.

The developed Matlab script was utilized to read the binary file of the oversampled master image and extracted high coherence pixel coordinates. A total of 748 pixel coordinates (red dots) that had high coherence values were obtained from the P platform and offshore support vessel (OSV) (see Fig. 3). However, 342 high coherence pixel coordinates on the OSV were discarded manually to avoid low correlation values due to the 
movements of the OSV, which was significantly influenced by temporal de-correlation effects. Thus, only 406 pixel coordinates on the $\mathrm{P}$ platform were utilized to facilitate the calculation of the offset vectors between the master and slave image pairs in the azimuth and range directions.

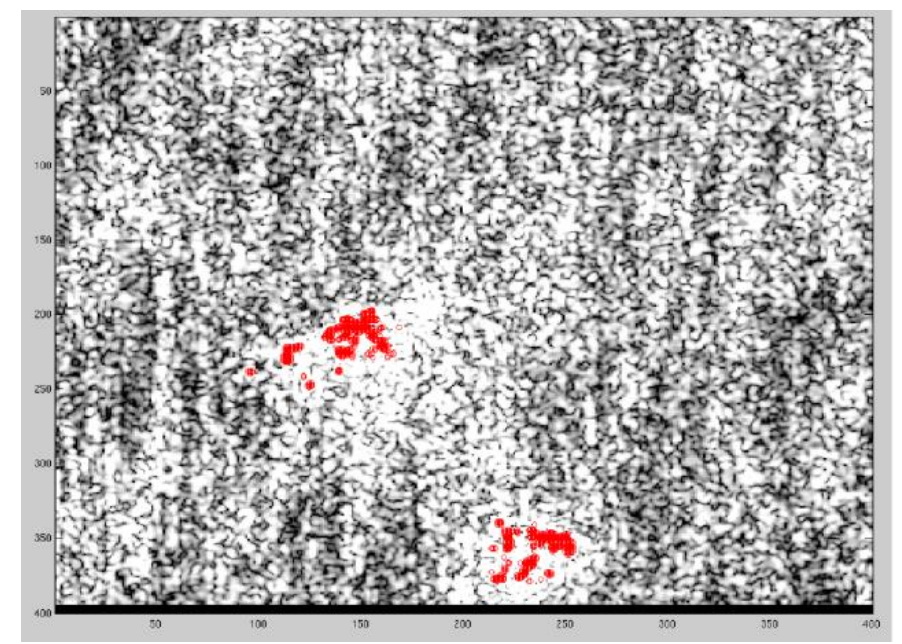

Fig. 3 Distribution of the high coherence pixels of the oversampled master image. The left side is the $\mathrm{P}$ platform while the right is the OSV.

\subsubsection{Interferograms Generation}

The co-registered images were processed to generate interferogram. The interferogram were formed by differencing the phase of every slave image to the phase of the selected master image. For N SAR images, N-1 inteferograms can be generated with respect to the master image. The interferogram phase has generally appeared as a series of fringes. One fringe represents a phase change of $2 \pi$ radians or half of the wavelength $\lambda / 2$ (e.g., $1.55 \mathrm{~cm}$ for TerraSAR-X) in surface displacement.

\subsubsection{Masking after a series of interferograms}

Masking has been developed using Matlab to remove the de-correlated area caused by the ocean. A matrix that had the same size (rows and columns) as the master image was created. The mask was created by assigning 0 to the matrix elements except for those of the offshore platform areas, where they were assigned 1. The master image in the binary format was opened and its pixels were multiplied with the created mask, and thus produced a new master image. The new master image was saved with the same name and format (binary) as the original master image. Fig. 4 shows the de-correlated area that has been masked, leaving the $\mathrm{P}$ platform to be involved for further processing. 


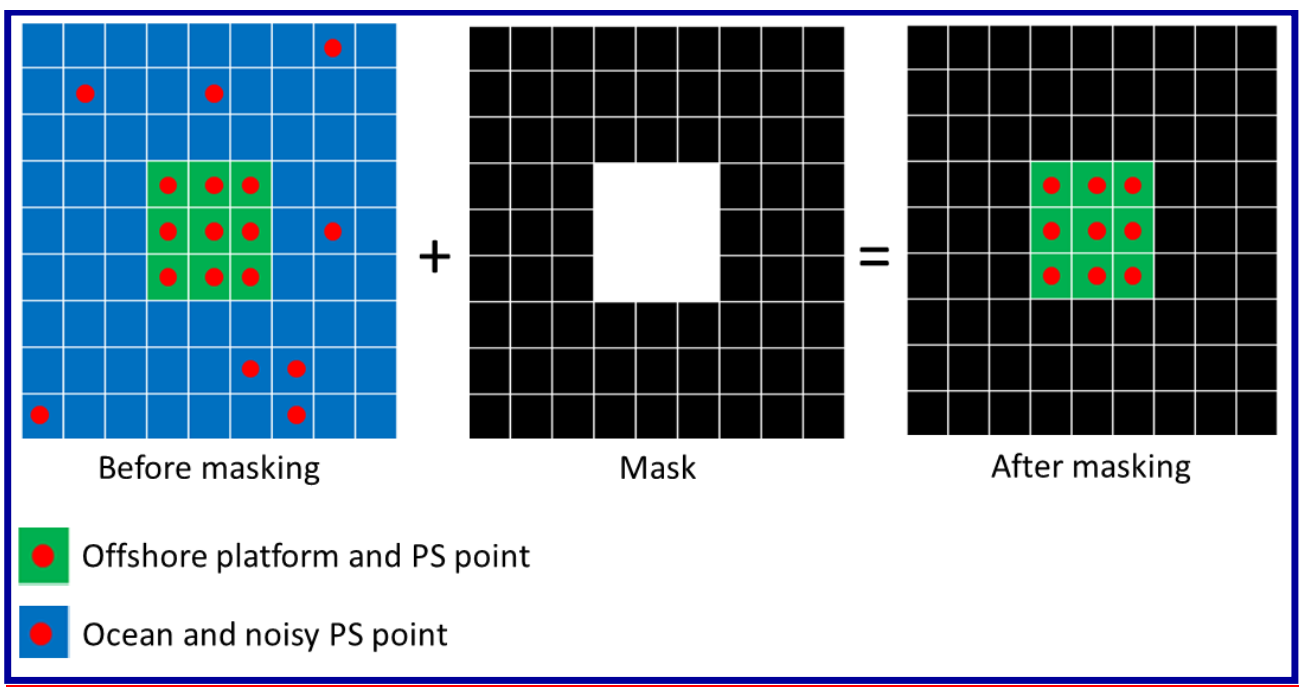

Fig. 4 Masking of the de-correlated area caused by the ocean, leaving only the P platform area.

\subsubsection{StaMPS technique}

The single patch interferogram has been utilized in this case as the study area was small, where only a small computational burden was needed. The initial selection of the PSCs was identified from the interferograms through the analysis of the amplitudes by setting the higher amplitude dispersion threshold value at 0.42 . The phase noise level for each candidate was estimated after removing the spatially correlated errors (i.e., deformation, atmosphere, orbital and part of the Digital Elevation Model (DEM) errors) and the spatially uncorrelated DEM error, and was then refined successively in a series of iterations. Temporal coherence was used as a measure of the phase noise/residual to reject any lowquality PSCs. Once the phase analysis for each pixel is done, the number of acceptable candidates was further dropped by allowing a maximum acceptable spatial density of 20 random phase pixels per $\mathrm{km}^{2}$.

The PS pixels that appear to be dominated by the adjacent pixels (side-lobe effects) and persist only in certain interferograms could be discarded. After that, the phase noise of the remaining candidates was compared with their neighbors (i.e., candidates which were connected by lines in the Delaunay network). Those pixels with a phase noise standard deviation smaller than the threshold were kept as the PS points; otherwise, they were considered as noisy pixels and were rejected. Also, the candidates with a noise level exceeding the weed maximum noise threshold were rejected. The spatially uncorrelated look angle error was estimated and subtracted from the wrapped phase before phase unwrapping. The spatially uncorrelated look angle error is caused by the difference between the assumed geometric center and the actual phase center for each pixel and can be estimated through its correlation with the perpendicular baseline.

The corrected phases of the PS points were filtered using an adaptive Goldstein filter in order to reduce its phase noise before unwrapping. It can then be unwrapped in 3D (two in space and one in time) to retrieve the absolute phase of the PS points. The phase differences between nearby PS points (over arcs) were first unwrapped in time under assumption that all phase differences lie between $-\pi$ and $\pi$. The unwrapped time series result was later filtered by low-pass filter to obtain smooth data. The outputs of the smoothed unwrapping in time were then used to construct the a priori probability density function (PDF) for the phase differences between PS points in every interferogram. By utilizing the derived PDF, 
the parameters of the cost function required for spatial unwrapping can be created. Then, the SNAPHU algorithm was applied for unwrapping the phase in space [17]. However, the SNAPHU cannot be directly applied to the sparse PS points and thus nearest-neighbour interpolation method was used to resample the irregular grid of the PS points to a regular grid. The results were first in each grid of unwrapped phase values and then the phase at the original PS points. The unwrapping error can be detected visually with the appearance of the phase jumps larger than $2 \pi$ between two adjacent pixels and it can be corrected by adding back or subtracting $n * 2 \pi$ manually to the badly unwrapped regions of PS points or just removing the badly unwrapped interferograms.

The unwrapped phase still contained the spatially correlated component of the look angle error although the study area was small. Hence, the error was estimated by spatial filtering of the unwrapped phase. However, the spatially correlated components of the atmospheric and orbital errors were not estimated in the processing as the errors were not expected to change within the small study area, and the errors were mostly subtracted by referencing the results of the mean phase value of the reference area. Finally, removing the spatially correlated errors left the phase due to the deformation signal and residual of spatially uncorrelated components of the DEM error which could be considered as noise. A detailed description of the StaMPS data processing can be referred to in [18 - 20].

\subsubsection{Geocoding}

The final output of the mean LOS deformation velocity value was extracted from the StaMPS and geocoded/displayed on the WorldView-2 satellite image using ArcGIS software.

\section{Results}

\subsection{Co-registration}

Fig. 5 shows the offset vectors (blue arrows) for the pixels that had a correlation higher than 0.6 which has been indicated by the size of the red circles. The top table in the figure shows several coherence pixel point numbers (red) and their corresponding offset vectors. The result shows that the length and direction of the offset vectors for the $\mathrm{P}$ platform were slightly different, indicating that the co-registration result was accurate and reliable. 


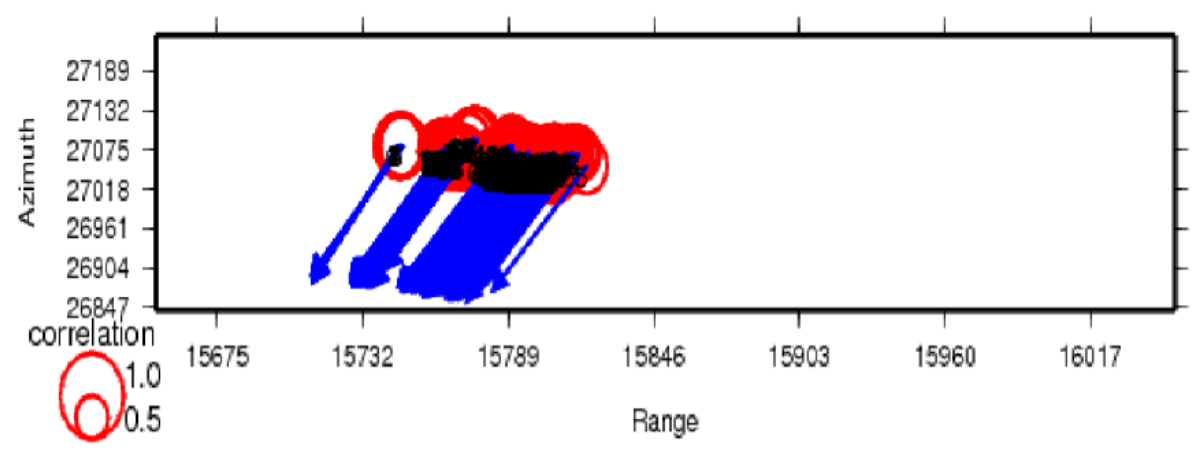

Fig. 5 Offset vectors between the master and selected slave images (25.08.2012) for the P platform.

The absolute error between the estimated and observed offset vectors in both the azimuth and range directions for all of the identified coherence pixels is shown in Fig. 6. From the histogram, it can be observed that most of the points had an absolute error less than a 0.1 pixel.
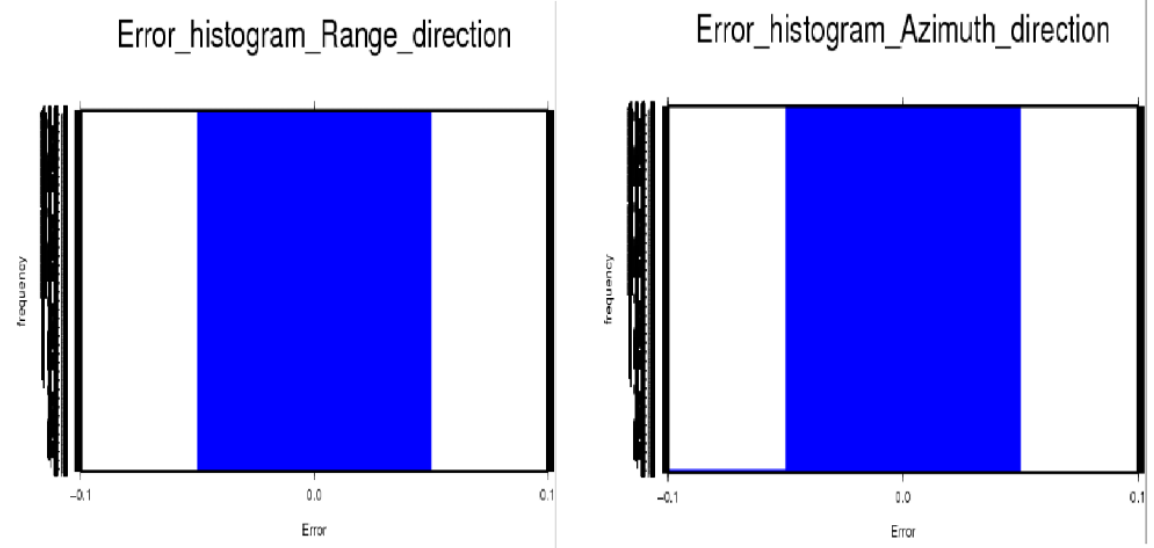

Fig. 6 Absolute error between the observed and estimated offset vectors in the azimuth and range directions for the 25.08.2012 slave image.

\subsection{Estimation of the Surface Deformation - Without Masking}

Fig. 7 shows some of the PS points that were observed at the ocean, which were not the reliable PS points. 


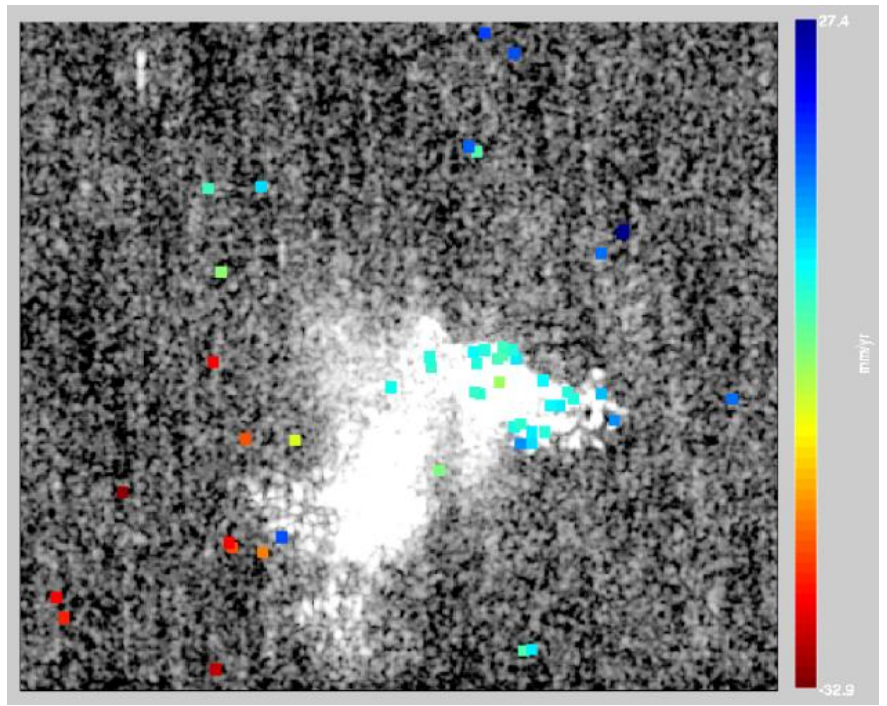

Fig.7 Without masking, and the weed standard deviation of 1.0 being applied.

Figs. 8 and 9 show that the PS points on the offshore platform were less dense when the standard deviation threshold used was lower than the suggested default value (1.0 radian) as an attempt to remove the noisy PS points located on the ocean.

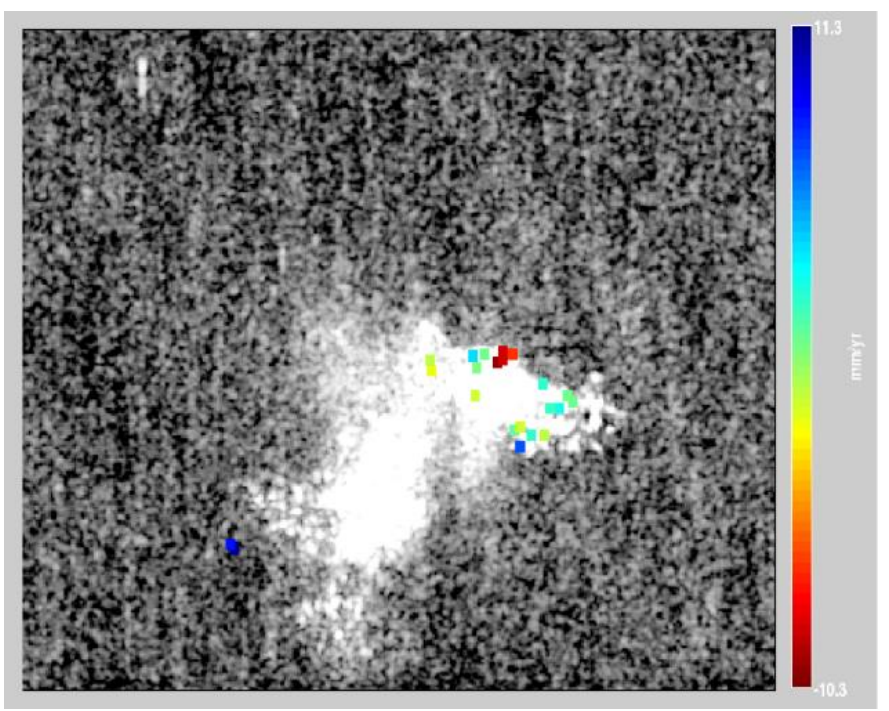

Fig. 8 When the weed standard deviation of 0.4 was applied, two noisy PS points were still located on the ocean. 


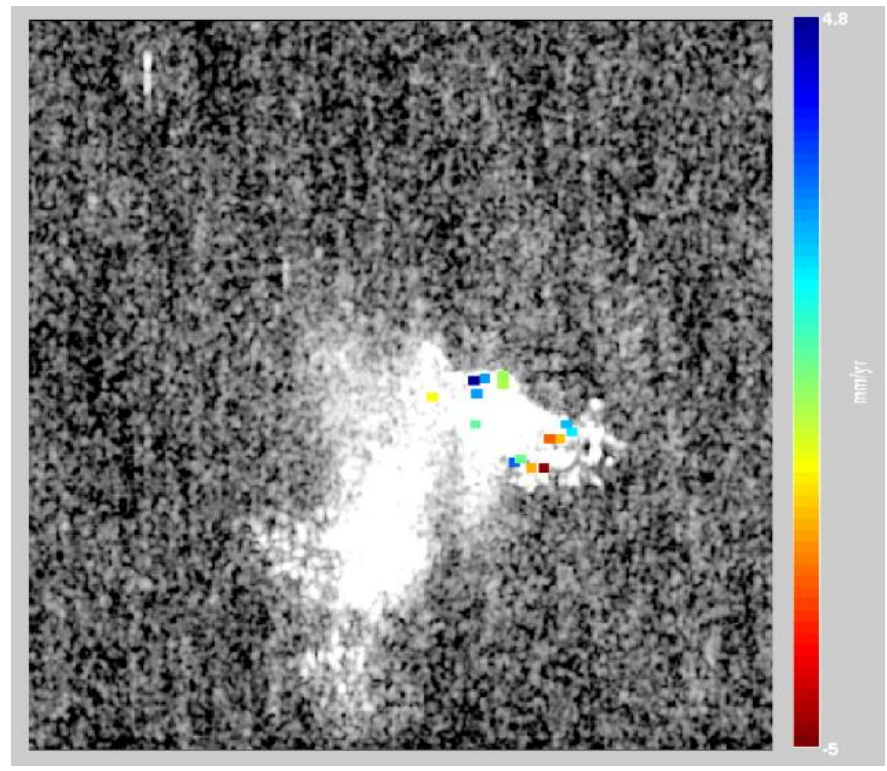

Fig. 9 When the weed standard deviation of 0.3 was applied, The noisy PS points had been removed but a lower number of PS points on the offshore platform can be observed.

Fig. 9 shows that only the dominant PS points were retained after the weed standard deviation of 0.3 was applied. However, lowering the weed standard deviation to 0.3 seemed to be excessive as the deformation signal was also lost, which caused the deformation to range from only -5.0 to $4.8 \mathrm{~mm} / \mathrm{y}$. With the impact of continuous waves and winds on the $\mathrm{P}$ platform, this small movement seemed very unreasonable.

The estimation of the standard deviation of the mean velocity per pixel was calculated to provide an indication of the precision for the mean velocity estimated. Fig. 10 shows the standard deviation of the estimated deformation rate when the weed standard deviation of 0.3 was applied, which ranged from 1.8 to $6.3 \mathrm{~mm} / \mathrm{y}$.

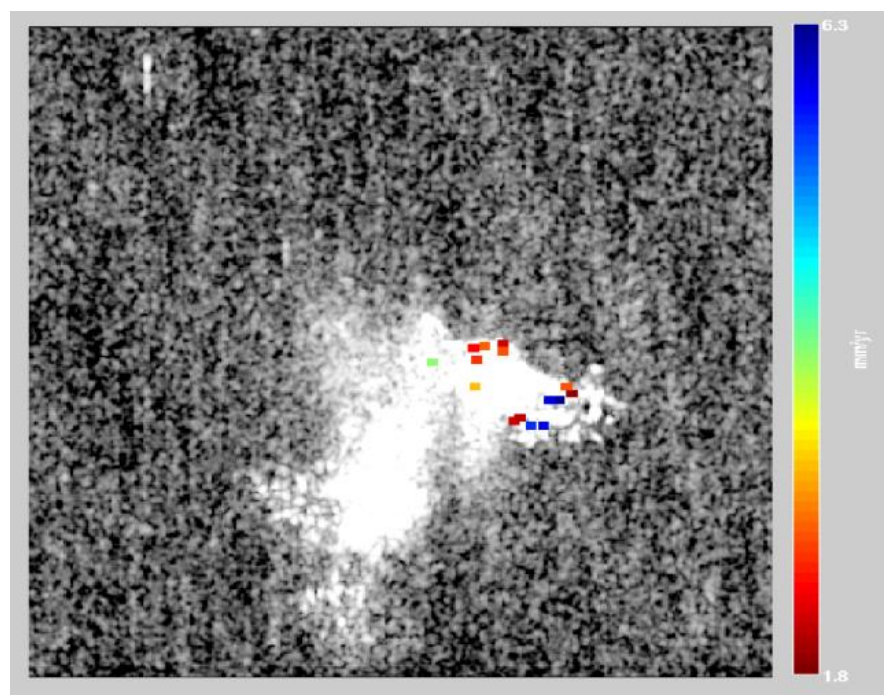

Fig. 10 Standard deviation of the estimated deformation rate when the weed standard deviation of 0.3 was applied. 


\subsection{Estimation of the Surface Deformation - After Masking}

An improvement of the spatial density PS points can be seen when masking has been applied and the slight relaxing of its selection criteria was performed by increasing the weed standard deviation to 1.0. Without using masking, the density of the PS points identified on the P platform was 15 points; but with the masking technique, 22 points were identified and the deformation signal had also been reinstated (see Fig. 11). Fig. 11 shows the velocity map of the P platform derived from the time series of 11 TerraSAR-X images during the period from August 2012 to April 2013. The colour levels of the PS points corresponded to the variation of the average deformation rates in the line of sight (LOS) direction which was from -5.6 to $10.5 \mathrm{~mm} / \mathrm{y}$, of which the red areas were affected by subsidence, while blue areas were uplift. The reliability of the identified deformation over the $\mathrm{P}$ platform can also be qualified based on the spatial pattern of the velocity of the available PS points (the smoother the pattern the better). The back side of the monitored platform experienced subsidence. The front side of the right portion of the platform experienced uplift, while the left side experienced subsidence.

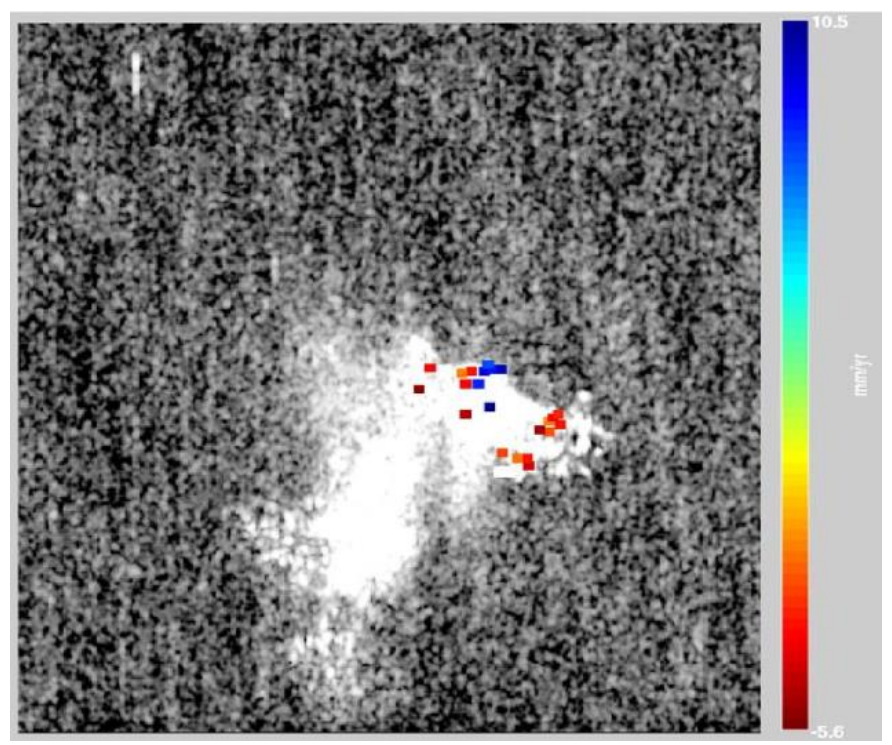

Fig. 11 When the weed standard deviation of 1.0 was applied together with masking.

Fig. 12 shows the standard deviation of the estimated deformation rate when the weed standard deviation of 1.0 was applied, which was from 1.5 to $5.1 \mathrm{~mm} / \mathrm{y}$. Thus, the small standard deviation indicated the small noise associated with each measurement. Moreover, the result shows that the weed standard deviation of 1.0 (with masking) was more precise than the weed standard deviation of 0.3 (without masking, which from 1.8 to $6.3 \mathrm{~mm} / \mathrm{y}$ ). 


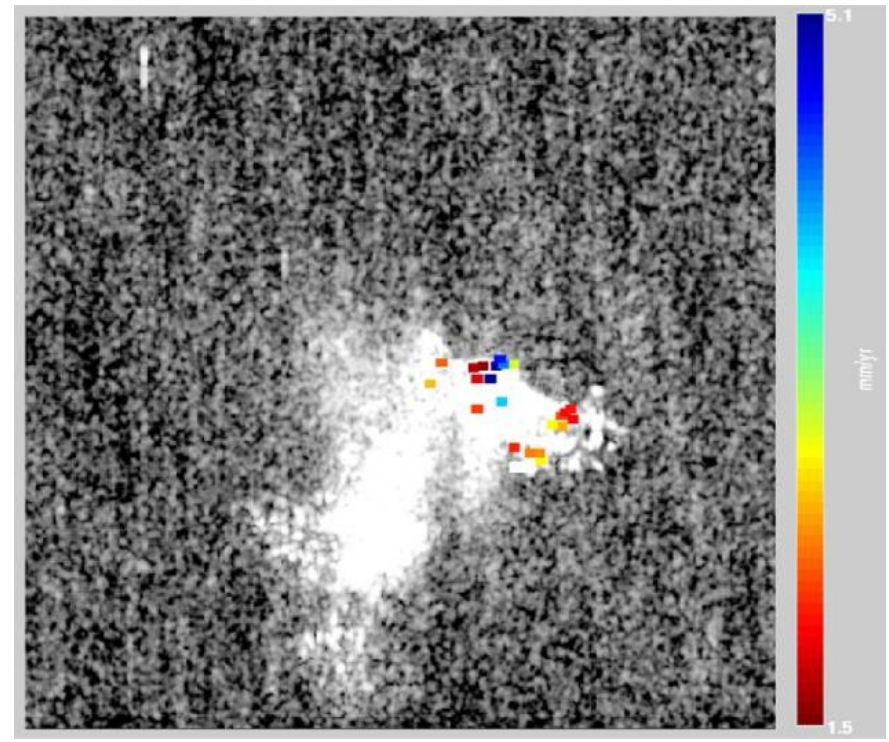

Fig. 12 Standard deviation of the estimated deformation rate when the weed standard deviation of 1.0 was applied together with masking.

Fig. 13 shows the estimated deformation history over the observed period for all 22 PS points. The time series showed small, nonlinear movements, which can be attributed to platform vibrations due to wind and waves. Moreover, due to the small number of scenes used in this study, the separation between the vibrations and permanent movement was difficult. Thus, long time series data was needed to identify the permanent movement/linear deformation. 


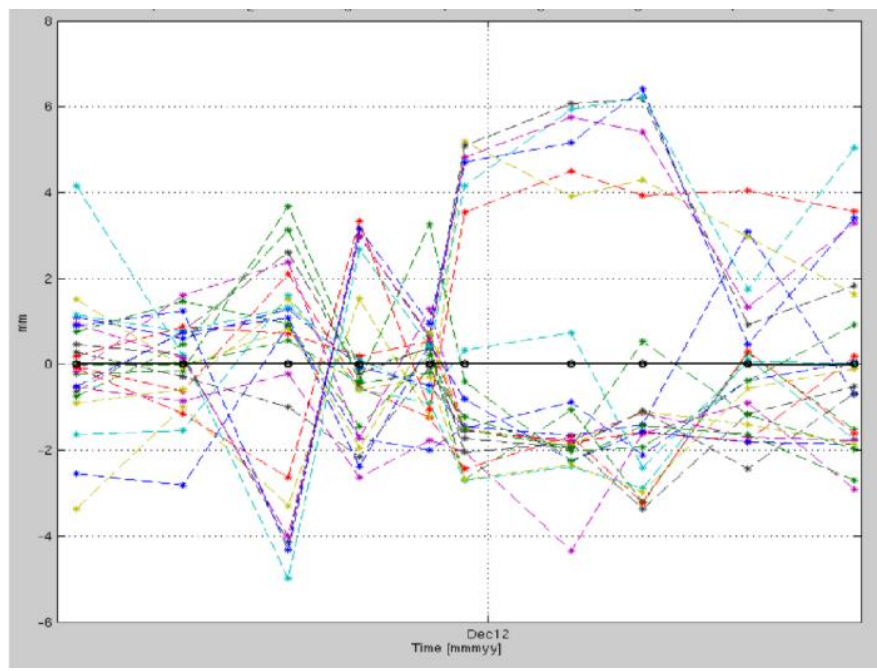

Fig. 13 Deformation time series of all of the PS points on the P platform. The specific date of the data acquisition can be referred in Table 1 .

\subsection{Geocoding}

The result of the deformation velocity map was superimposed over a high resolution WorldView-2 optical image $(0.5 \mathrm{~m})$ as shown in Fig. 14. With the high resolution WorldView-2 optical image utilized in this study, the individual structures that were affected from the deformation were identifiable. The color of each PS point indicates the relative phase difference with respect to the mean reference point.

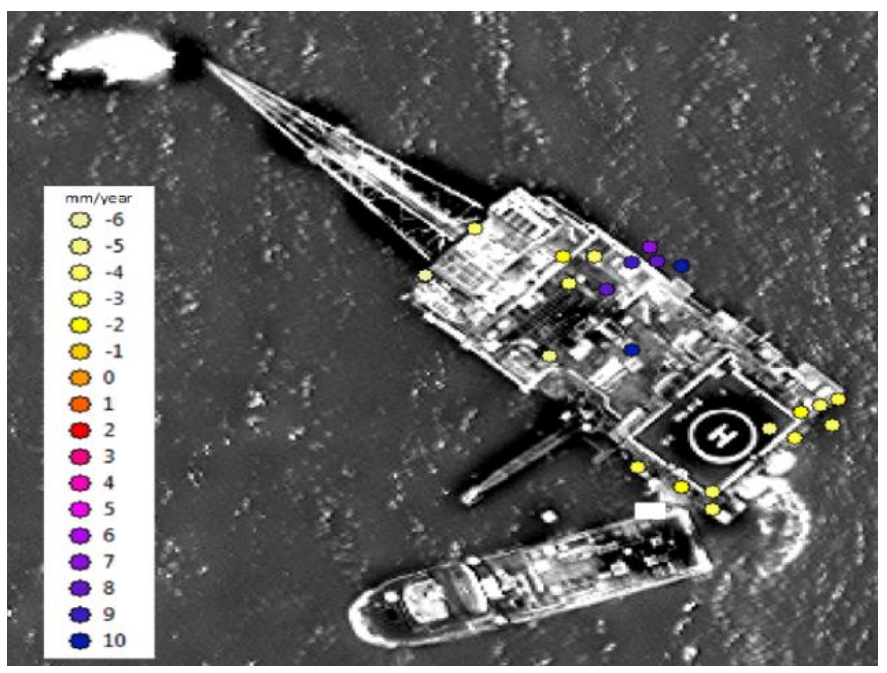

Fig. 14 Deformation velocity map superimposed on the WorldView-2 image of the P platform. Note that the TerraSAR-X radar image and WorldView optical image have slightly different geometries. 


\section{Discussions}

This section will discuss the limitations of and possible solutions for the PSI technique on the offshore platform deformation monitoring analysis.

\section{Co-registration problem}

The co-registration was fairly difficult as the monitored offshore platform was a small structure and most of the area in the image contained an uncorrelated area of the sea. Thus, the co-registration could be applied to only this small area, with no possibility of monitoring the movement of the platform with respect to another, distant object, such as land or another platform.

\section{Detection of only small movements}

The PSI technique is ambiguous, by principle, due to the fact that the phase is measured only in the $(-\varphi, \varphi)$ interval, instead of the (unambiguous) distance. Therefore, an arbitrary integer multiple of $2 \varphi$ (or $\lambda / 2)$ can be added/subtracted from the measurement in every acquisition time. This ambiguity limits the InSAR/PS applications to cases with slow deformations, where the difference of the position between the subsequent acquisition times is significantly lower than $\lambda / 4$. For an X-band image, $\lambda / 4$ corresponds to $7.75 \mathrm{~mm}$ (in LOS). If the integer multiple of even one of the measurements is estimated in a wrong way (i.e., not corresponding to reality), all of the other measurements are often biased. As the offshore platform is located on the ocean, the movement/vibration of the platform due to strong winds and waves can easily exceed the $\lambda / 4$. Thus, higher wavelength data from the C-band or L-band are considered to be used but the L-band data might not be suitable as it is less sensitive to the movements (due to the long wavelength).

\section{Low number of images and short period of observation times}

The low number of images and short period of observation time were unable to capture the offshore platform deformation: the results obtained show that only small movements and non-linear time series can be observed in this case. The small movement and non-linear time series are expected to have been caused by the vibrations of the monitored offshore platform. Thus, the number of images should be significantly increased, together with a longer period of time to identify the permanent movement, and separate it from the vibrations.

\section{Selection of the reference point and relative measurement}

In this study, the reference point was also located on the platform and, therefore, only the relative movement of the platform could be determined (e.g., east side with respect to the west side). The movement of the platform itself could not be monitored with respect to other platforms, islands or continents. The enlargement of the processing area to include such objects will have a problem during the image co-registration, as mentioned before, due to the de-correlation of the sea area. Moreover, the distance to such objects is longer than a few kilometers from the monitored offshore platform, and therefore, the atmospheric delay has to be dealt with, expecting significantly higher values above the sea than above the ground. Thus, to know the absolute movement of the points on the platform, the movement of the reference point should be monitored using the GNSS observation on every InSAR acquisition image to know, very well, how the reference point moves. At the end, the 
deformation results can be added to the movement of the reference point measured by the GNSS technique for the correction.

\section{Conclusions}

This study has demonstrated the first experience on monitoring the movement of an offshore platform using the PSI technique. The co-registration of the offshore platform was successfully implemented by extracting high coherence pixels. By applying masking as the new module proposed in this study, the noisy PS points on the ocean were removed and the number of the PS points increased from 15 to 22 points and a smoother pattern of the velocity estimation could be seen. Besides that, the result was more precise when applying the weed standard deviation of 1.0 (with masking) compared with the weed standard deviation of 0.3 (without masking). This study has shown that the monitored platform had maximum subsidence/downward movement and uplift/upward rates of -6 to $11 \mathrm{~mm} / \mathrm{y}$, respectively. The small standard deviation values of the estimated deformation, which ranged from 1.5 to $5.1 \mathrm{~mm} / \mathrm{y}$, indicated that the results were accurate and reliable. However, a significantly higher number of images (together with a significantly longer monitoring period) would be required to confirm such values, to reliably separate the permanent movement from the vibration component (caused by wind and waves) and possibly to help identify possible unwrapping errors. Some limitations of and possible solutions for the PSI technique were highlighted that may help future users to choose the most appropriate way for monitoring offshore platforms. The potential of the PSI technique for deformation monitoring of offshore platforms can be further enhanced with the PSI result validations. The result validations are useful to obtain an assessment of the sensitivity, precision and accuracy of the PSI technique.

This research was supported by the Yayasan Universiti Teknologi Petronas - Fundamental Research Grant (YUTP-FRG) (0153AA-H15). The authors wish to thank Mrs Ivana Hlavacova from GISAT, Czech Republic, for her extensive help in editing and interpreting the results of this study.

\section{References}

1. May, P., Sanderson, D., Sharp, J., \& Stacey, A. Structural integrity monitoring: Review and appraisal of current technologies for offshore applications. Paper presented the ASME 2008 27th International Conference on Offshore Mechanics and Arctic Engineering (2008).

2. Masnan, M. N. Best Practice for Offshore Platform Deformation Survey Using Global Navigation Satellite System. Master dissertation, Universiti Teknologi Malaysia (2015).

3. N. Widjajanti and A.N. Matori. Evaluation of GPS data for offshore platform subsidence. $7^{\text {th }}$ Asia Pacific Structural Eng. And Construction Conf. (APSEC 2009) \& $2^{\text {nd }}$ European Asian Civ. Eng. Forum (EACEF 2009), Langkawi, Malaysia (2009).

4. Setan, H., \& Othman, R. Monitoring of offshore platform subsidence using permanent GPS stations. Positioning, 1(10), 0 (2006).

5. Song, Y., Yu, Y., Zhang, C., Dong, W., \& Ou, J. Design of self-contained sensor for monitoring of deep-sea offshore platform. Paper presented at the Sensors and Smart Structures Technologies for Civil, Mechanical, and Aerospace Systems 2013 (2013).

6. Tang, D., Deng, M. L., He, F., \& Yue, Q. J. A Newly Developed Self-Contained Inclinometer for Offshore Platform Anchor Monitoring. Paper presented at the Advanced Materials Research (2013).

7. Ren, L., Li, H., Zhou, J., Li, D.-S., \& Sun, L. Health monitoring system for offshore platform with fiber Bragg grating sensors. Optical Engineering, 45, 8, (2006). 
8. Sousa, J. J., Hlavacova, I., Bakon, M., Lazecky, M., Patricio, G., Guimaraes, P., Ruiz, A.M., Bastos, L., \& Sousa, A. Potential of Multi-Temporal InSAR Techniques for Bridges and Dams Monitoring. In: SARWatch Workshop, CENTERIS 2014, Troia, Portugal, 15 -17 October (2014).

9. Qin, Y., \& Perissin, D. Monitoring Ground Subsidence in Hong Kong via Spaceborne Radar: Experiments and Validation. Remote Sensing 7:10715-10736 (2015).

10. Milillo, P., Perissin, D., Salzer, J. T., Lundgren, P., Lacava, G., Milillo, G., \& Serio, C. Monitoring dam structural health from space: Insights from novel InSAR techniques and multi-parametric modeling applied to the Pertusillo dam Basilicata, Italy. International Journal of Applied Earth Observation and Geoinformation, 52, 221-229 (2016).

11. Bekaert, D. P. S. Interferometric Synthetic Aperture Radar for slow slip applications. $\mathrm{PhD}$ dissertation, University of Leeds (2015).

12. Jónsson, S. Modeling volcano and earthquake deformation from satellite radar interferometric observations. PhD dissertation, Stanford University (2002).

13. Matori, A. N., Latip, A. S. A., Harahap, I. S. H., \& Perissin, D. Deformation Monitoring of Offshore Platform Using the Persistent Scatterer Interferometry Technique. Applied Mechanics and Materials 567:325-330 (2014).

14. Kampes, B.M., Hanssen, R.F., Perski, Z. Radar interferometry with public domain tools. In: Proceedings of FRINGE 2003, Frascati, Italy (2003).

15. Hooper, A. A multi-temporal InSAR method incorporating both persistent scatterer and small baseline approaches. Geophys. Res. Lett. 35 (16) (2008).

16. Hooper, A, \& Zebker, H.A. Phase unwrapping in three dimensions with application to InSAR time series. JOSA A 24 (9), 2737-2747 (2007).

17. Chen., C.W. Statistical-cost network-flow approaches to two-dimensional phase

18. unwrapping for radar interferometry. PhD thesis, Stanford University (2001).

19. Hooper, A. Persistent Scatterer Radar Interferometry for Crustal Deformation Studies and Modeling of Volcanic Deformation. Ph.D. thesis, Stanford University (2006).

20. Sousa, J.J. Potential of Integrating PSI Methodologies in the Detection of Surface

21. Deformation. PhD Thesis, University of Porto, Portugal (2009).

22. Latip, A. S. A., Matori, A. N., Aobpaet, A., and Din, A.H.M. Monitoring of Offshore Platform Deformation with Stanford Method of Persistent Scatterer (StaMPS). The 2015 International Conference on Space Science and Communication (IconSpace2015). Langkawi, Malaysia (2015). 